\title{
Unified dispersion model for the electric susceptibilities of physically realizable materials
}

\author{
Richard A. Shepherd, Sr. \\ Rolls-Royce North American Technologies, Incorporated, Rolls-Royce, 450 South Meridian Street, Indianapolis, Indiana 46225, USA
}

(Received 21 December 2018; published 15 May 2019)

\begin{abstract}
Based on the powerful method of network control theory, a complete, classically derived, broadband, analytic dispersion model is presented which describes electric susceptibility for a rigorously physically realizable, dielectric material system with a possible dc conductivity contributor. The analysis results in a susceptibility model constructed entirely from pole-residue groups. As a result, solitary Debye-type poles are not physically supported in the broadband analytic dispersion theory presented in this paper. While previous investigators have focused primarily on modeling susceptibility using only negative complex-conjugate poles, the type found naturally at optical frequencies and above, this analysis was conducted under no such restriction; hence, it is considered unified and should apply to all classical-type susceptibility behavior at all frequencies from dc to daylight, including some of the ultraviolet region where the material-continuum assumption begins to break down. Excluding conductivity due to the absence of any proof of magnetic monopole existence, the model should be equally applicable for modeling magnetic susceptibility dispersion. Based upon a rigorous emphasis on physical realizability, the complete, classical dispersion model is demonstrated by successfully modeling the complex permittivity of liquid water at $25^{\circ} \mathrm{C}$ using measurement data from $30 \mathrm{MHz}$ to $1200 \mathrm{THz}$.
\end{abstract}

DOI: 10.1103/PhysRevA.99.053822

\section{INTRODUCTION}

Water is arguably the most important chemical compound to carbon-based life as we know it. It covers $70 \%$ of the surface of the Earth, and according to Utz [1] as a free compound it makes up approximately $60 \%$ of our adult bodies. Modeling the spectrum of water in the 1960's was important for studying a burgeoning topic we now refer to as climate change. Today it is more important than ever in the relatively new scientific branches of biophysics and bioengineering due to the interest in modeling living tissue and its responses to electromagnetic radiation in both the detection and the treatment of disease. An excellent example of the former can be found in the pioneering work of Subramanian et al. [2], who have been developing methods for optically measuring nanostructural deviations in cells for the purpose of detecting cancer much earlier than any conventional imaging technique. Since cells are primarily made of water, the team is constantly modeling water and since their primary analysis method is the finite-element time domain, they need to include water's broadband dispersion into their models.

Although this investigator has been unable to find direct evidence, it is hard to imagine that the optical (infrared and visible) spectrum of such an important compound would not have first been recorded shortly after the discovery of infrared radiation by the famous astronomer William Herschel in the year 1800 [3]. We know that by 1840 [4], his son,

Published by the American Physical Society under the terms of the Creative Commons Attribution 4.0 International license. Further distribution of this work must maintain attribution to the author(s) and the published article's title, journal citation, and DOI.
John Herschel, was photographing Fraunhofer lines well into the infrared, lines which include thousands of rotational and vibrational absorptions from atmospheric water vapor. During this investigation, tables were encountered which included liquid water permittivity values measured as far back as 1895 [5] clearly showing the occurrence of a dramatic absorption minimum occurring in the ultraviolet-visible spectrum and over seven orders of magnitude lower than the measured absorptions of the $v_{1}$ and $\nu_{3}$ IR band heads. Clearly, knowledge of water's mysterious optical transparency valley has existed for well over 100 and probably closer to 200 years.

Of course, before we can model the spectrum we need to measure the spectrum or at least have reliably measured data. It is estimated there are over 1000 published papers reporting the measured spectrum of water in some region of the electromagnetic spectrum, and perhaps only 20 totalspectrum models based upon compilations of the measured data. The common element of all these compilations is that the authors each separated the spectrum into several regions, usually six, seven, or even nine, and used purely empirically derived fitting functions, tailored and limited to each region. Given the importance of water and all the scientific curiosity in its remarkable spectrum, it is difficult to believe that we are basically left with only data tables from which to consult for this information.

From a literature search only two papers were encountered where the investigators attempted to model the complete spectrum of water based entirely on physics, one in 1997 by Diaz and Alexopoulos [6] and the latest one in 2007 by Shubitidze and Osterberg [7]. The former can be considered a landmark paper because Diaz and Alexopoulos showed us that for materials whose macroscopic response can be described through classical equations of motion, the electric susceptibility should be completely representable as a rational 
function and thus reopened our eyes to a more thorough understanding of modeling dispersion through the manipulation of poles and zeros just as Berreman and Unterwald [8] first showed us for $\mathrm{PrCl}_{3}$ and $\mathrm{LaCl}_{3}$ in 1968 . However, as opposed to the permittivity, Diaz and Alexopoulos wisely chose to model the susceptibility. An even wiser decision was that they chose to model it for water. Ultimately, due to water's importance, popularity, and bewildering spectrum, the team could not have selected a better testbed on which to demonstrate their proposed improvement to classical dispersion theory. Although their simulated spectrum appears to have significant discrepancies $>100 \%$ in some regions, the fact that Diaz and Alexopoulos were able to produce an optical valley for water better than anyone previously made their paper one of the most talked about in the late 1990's.

Publishing their own model 10 years later, the team of Shubitidze and Osterberg [7], with the exception of a couple of spurious peaks, was able to accurately reproduce the water spectrum data compiled in 1981 by Segelstein [9] with an impressive maximum deviation of just $10 \%$. However, due to the distinct lack of fanfare in the decade since it was published, it appears that their model may have fallen short of the scientific community's expectations. Because their model includes factoring Fermi-Dirac statistics with the Debye and Lorentzian responses, it might be considered too complicated to be practical. Some may even reject the model simply because the Fermi-Dirac distribution function does not possess the correct symmetry for causality, but this is a common problem encountered (and almost always ignored) in quantum mechanics. The model could also be faulted for the fact that the rotational, vibrational, and vibronic regions are not unified, even though the team resorts to using quantum mechanics. In general the paper does not appear to be proposing any improvement to dispersion theory, but their model does provide a significantly better fit for water than the one by Diaz and Alexopoulos [6].

Although a prime motivator is to derive a complete analytic model for the electric susceptibility of water, the approach will not be so limited. The intent and ultimate goal in this work is to cast a wide net, and derive a model which encompasses all physically realizable materials. Under the assumption that liquid water is such a material, i.e., that it behaves classically in the frequency range of $\mathrm{dc}$ to $\mathrm{UV}$, the water dispersion model will be obtained when such a universally applicable model is successfully derived.

Complex analytic function continuation theory has helped increase the knowledge of electric susceptibility dispersion through mathematical models which expand understanding into solution spaces where measured data, for physical reasons, are nonexistent. Continuation theory also allows us the ability to estimate dispersion behavior in the time domain, based simply on measurements at just a handful of frequencies. Because time-domain electromagnetic computation methods determine responses to short-pulse stimuli, they need broadband, compact mathematical models to accurately account for dispersion. Truly broadband analytic functions which are compact in both the time domain and the frequency domain neatly fit the bill. Only analytic functions can supply the real and imaginary responses in both time and frequency domains, using the same set of parameters. Clearly, a good analytic model will save countless hours of measurement time, simulation programming time, and runtime.

Most readers at some time have likely been exposed to a modern derivation of the famous Kramers-Kronig (KK) relations between real and imaginary electric permittivity, based upon a few physical assumptions, which are that the response function is

(1) Mathematically representable in an analytic functional form, basically the inherent assumption in any physical science course involving differential equations;

(2) Linear with respect to a stimulus force, thereby transformable and linear with respect to superposition; i.e., the sum of the transforms is equal to the transform of the sum;

(3) Analytically continuable in the complex frequency plane, also known as the complex $\omega$ plane; the response function can be represented in the complex plane as long as it smoothly delivers physically realizable results whenever the frequency or time variable approaches real values; and most importantly,

(4) Observable in the time domain; the response function must be physically realizable. Therefore, it must be causal and real in the time domain; the function must be stable and dissipate energy, not amplify.

Based upon these assumptions, generations of investigators [10] have derived mathematical models for what are considered Kramers-Kronig compatible response functions, the most popular of which are the Drude, the Debye, the modified Debye, and the Lorentzian. These models have enjoyed immense popularity and success at accurately predicting dielectric behavior, with perhaps one exception, the Debye.

According to Heileman et al. [11] the most utilized models in the simulation of electromagnetic fields at the biological cellular level are the Debye and the Cole-Cole [12] models, in spite of the fact that the Debye model has more than a few shortcomings:

(1) In the time domain, the Debye model is discontinuous at time $t=0$. The time-domain kernel is zero at $t<0$ and not zero at $t \geqslant 0$. This is just not physically realizable.

(2) At increasingly high frequencies, under the Debye model, the complex susceptibility value is predicted to fall off inversely as $\omega$ but instead it has been observed for virtually all materials to fall off inversely as $\omega^{2}$, and as reported in 1932 by Van Vleck [13], the steeper rate is also supported using quantum mechanics.

(3) The dc conductivity value is predicted under the Debye model to be a constant with respect to frequency, yet in 1900, eight years before Peter Debye even earned his doctorate, Paul Drude [14] had already published, in a book, a conductivity model still in use today, showing that conductivity has a distinctly Debye-type dispersion behavior of its own (obviously it was not referred to by that name at the time).

(4) Another major flaw shows up when the Debye model is applied to the magnetic susceptibility of magnetic materials; often the measured real component turns out to be undeniably less than zero which should never happen under the Debye model since the slope is negative for all $\omega$ and therefore has no mathematical mechanism to return to zero as $\omega \rightarrow \infty$.

(5) Debye susceptibility behavior appears to be only observable at radio frequencies (rf), never optical. So, in spite of 
its success at radio frequencies, there is definitely something incomplete with the Debye model.

In 1968 Berreman and Unterwald [8] published a paper showing a more powerful derivation of a mathematical model for electric permittivity could be made using the four assumptions enumerated earlier, yet without assuming a physical model a priori, but instead a mathematical model based on analytic continuation and network control theory in which the investigator can adjust poles and zeros to simulate the measured response. They considered the conventional practice of modeling the response as uncoupled, harmonically damped oscillators "overly restrictive" when compared to the analytic approach.

Since electric permittivity and susceptibility differ only by a constant, we are able to represent the susceptibility by a rational function of the same form used by the authors in [8] for representing the permittivity in their paper, i.e.,

$$
\chi(\omega)=\prod_{m=1}^{M}\left(\omega-Z_{m}\right) / \prod_{n=1}^{N}\left(\omega-P_{n}\right),
$$

where $\chi(\omega)$ is the electric susceptibility relative to $\varepsilon_{0}$, the permittivity of free space; $Z_{m}$ refers to the system zeros; and $P_{n}$ refers to the system poles. Diaz and Alexopoulos [6] argue that for physically realizable materials we should be able to exclude essential singularities and branch points from our response function, leaving only zeros and poles, therefore meeting all the necessary requirements to be completely represented by a rational function of the form expressed in (1). This is probably the most important result in their paper.

At this point of the derivation Diaz and Alexopoulos [6] employ partial fraction decomposition; however, in the resulting equation the poles are expressly restricted to being negative complex-conjugate pairs, despite the fact that purely imaginary poles are also allowed. By formally excluding such a large class of poles from the resulting equation as well as their mathematical proofs, statements to the contrary notwithstanding, the duo's resulting mathematical model and analysis cannot be considered complete.

It is worth pointing out that if the complete electric susceptibility system is representable by the rational function in (1), the popular Cole-Cole [12] and Havriliak-Negami [15] models, with their fractional exponents, must be incomplete. It is also worth pointing out that to be physically realizable according to network theory, the resulting coefficients of the polynomials in both the numerator and the denominator in (1) are purely real for powers of $(i \omega)$, not $\omega$, which, as revealed later in the paper, is the reason that complex poles will not appear as complex-conjugate pairs.

In this paper the investigation will begin as in Berreman and Unterwald's [8] paper and explore the implications of modeling physical phenomena by using complex physical network control theory. As opposed to their pole-zero approach, the investigation will be switched to a more physically insightful pole-residue approach, and will not be confined to frequencies in just the infrared spectral domain. In further contrast to the previous works $[6,8]$, the scope of the formal development will include poles on the imaginary $\omega$ axis. It is the author's intent that this theory be universally applicable at any frequency for any material which exhibits classically macroscopic responses to electromagnetic fields.

\section{MODEL DERIVATION}

\section{A. Partial fraction decomposition}

To be Kramers-Kronig compatible, the susceptibility function in (1) must have more binomials in the denominator than in the numerator; therefore, it must be true that $M<N$. This permits us to perform partial fraction decomposition for susceptibility. For simplicity, let us assume at this time that all the poles are simple, i.e., no duplicates. The result is

$$
\chi(\omega)=\sum_{n} \frac{i \sigma_{n}}{\left(\omega-P_{n}\right)},
$$

where the $\sigma_{n}$ 's are as yet undetermined parameters (referred to as residues) from the partial fraction decomposition.

When transformed into the time domain, the Debye dispersion functions which appear on the right yield

$$
\chi(t)=u(t) \sum_{n} \sigma_{n} \exp \left(-i P_{n} t\right)
$$

where $u(t)$ is the Heaviside step function [16] equal to 0 for $t<0,1$ for $t>0$, and for slope continuity, 0.5 at $t=0$.

Following the analysis by Jackson [17], some insight into the relationship of $\chi(\omega)$ with the susceptibility around $t=$ $0^{+}$can be gained by conducting integration by parts on the Fourier transform of (3), resulting in the asymptotic series:

$$
\chi(\omega) \simeq \frac{i \chi\left(t=0^{+}\right)}{\omega}-\frac{\dot{\chi}\left(t=0^{+}\right)}{\omega^{2}}+\cdots .
$$

While the functions in (3) might indeed be analytic, Jackson notes that the first term in (4) leads to the "unphysical" implication that $\chi\left(t=0^{-}\right)=0$, but $\chi\left(t=0^{+}\right)$might not $=$ 0 . Based on this, Jackson concludes that "the first term in the series is absent and that $\varepsilon(\omega)-1$ " [i.e., $\chi(\omega)$ ] "falls off at high frequencies as $\omega^{-2}$." In other words, for the model to be physically realizable, it is absolutely required that $\chi(t=$ $\left.0^{+}\right)=0$. According to (3), this leads us to the following sum rule over all the residues:

$$
\sum_{n} \sigma_{n}=0 .
$$

It also leads us to the conclusion that $M=N-2$ in (1). It is comforting to know that the assumption that $M<N$ is self-consistent and even more, that for physically realizable materials, susceptibility falls off one whole degree faster than is minimally required to be Kramers-Kronig compatible.

\section{B. Grouping poles}

Let us assume for the sake of the practicality of this model that we can subdivide the poles into small independent groups, one for each observable transition. For a group of poles to be considered independent from other groups for all $t \geqslant 0$, the analytic representation for the group itself must be physically realizable; otherwise, it would be dependent on poles outside the group to bring the total representation back into being physically realizable, thus violating the independence assumption. 
To formally force the susceptibility to be zero at $t=0^{+}$ for an isolated pole in (3), $\sigma_{n}$ by itself would have to be equal to zero, but this would be a trivial solution. From this we can conclude that for a material system to be physically realizable, single poles expressed in (2) or (3) are forbidden as independent entities; instead, they must interact with other poles. At a minimum, poles must appear in pairs; however, note that while this is sufficient it is not a necessary condition for (5) to be true, but merely the simplest grouping possible.

With no further suggestion as to specific grouping, this model is a complete model. Since the Kramers-Kronig relations are considered complete and the rational functional form for susceptibility in (1) is considered complete, then so must the mathematically equivalent pole-residue form in (2) be considered complete, at least for any material system which can be described using simple poles, which in the real world should mean any physically realizable material; after all, measuring a perfectly modified Debye transition has proved elusive. Thus far the assumptions are so basic as to imply that the permittivity of virtually any physically realizable material should fit this model over several transitions, and frequency bands as long as the laws of classical physics apply to the material system.

\section{Pole pairs}

Let us explore the consequences of a susceptibility model where each transition can be described exclusively by pole pairings. These pairings can be expressed mathematically in both frequency and time domains, respectively, by

$$
\chi(\omega)=\sum_{n} \frac{i \sigma_{1, n}}{\left(\omega-P_{1, n}\right)}+\frac{i \sigma_{2, n}}{\left(\omega-P_{2, n}\right)},
$$

and

$$
\chi(t)=u(t) \sum_{n} \sigma_{1, n} \exp \left(-i P_{1, n} t\right)+\sigma_{2, n} \exp \left(-i P_{2, n} t\right)
$$

under the independence-based requirement that $\sigma_{2, n}=-\sigma_{1, n}$. Applying this condition, the susceptibility in (6) can now be expressed as a sum of complex Lorentzians.

$$
\chi(\omega)=\sum_{n} \frac{i \sigma_{1, n}\left(P_{1, n}-P_{2, n}\right)}{P_{1, n} P_{2, n}-\left(P_{1, n}+P_{2, n}\right) \omega+\omega^{2}} .
$$

At first glance this form appears to suggest that the poles must be simple poles, else the numerator must yield a trivial contribution; however, this is completely due to the inherent assumption we made at the beginning that all the poles are simple, an assumption which was made only to simplify the derivation. The question as to the possible existence of duplicate poles will be addressed in section $E$.

To gain a better understanding of the constraints on (8), we shall apply the causal-based symmetry requirement, $\chi(\omega)=$ $\chi^{*}\left(-\omega^{*}\right)$. By comparing like powers, the result of the symmetry requirement yields the following realizations:

(1) For coefficients of the odd powers of $\omega$, the real components must be zero.

(2) For coefficients of the even powers of $\omega$, the imaginary components must be zero.
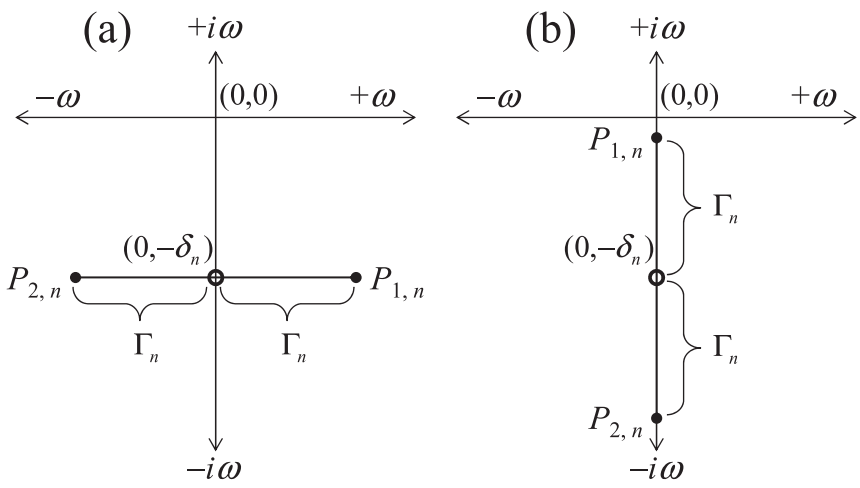

FIG. 1. Complex $\omega$-plane diagrams showing the two types of acceptable pole pairs: (a) negative complex conjugates, and (b) purely imaginary.

The above realizations lead to three results:

$$
\begin{gathered}
\operatorname{Re}\left(P_{1, n}+P_{2, n}\right)=0, \\
\operatorname{Im}\left(P_{1, n} P_{2, n}\right)=0,
\end{gathered}
$$

and

$$
\operatorname{Im}\left[i \sigma_{1, n}\left(P_{1, n}-P_{2, n}\right)\right]=0
$$

It is now possible to assess the types of poles that are permissible in this model. The two types of allowed poles are illustrated in Fig. 1. To no surprise, (9) and (10) are clearly satisfied using negative complex-conjugate paired poles such as the ones illustrated in Fig. 1(a). However, they are also satisfied using purely imaginary paired poles which are illustrated in Fig. 1(b); one of the poles can even be equal to zero, giving rise to the sole Drude component in this model. Further, according to (11), all the numerators in (8) are real. In short, the analytical result of causality is that the coefficient in front of the $\omega$ term is purely imaginary, while the coefficients for the other two powers of $\omega(0$ and 2$)$ are purely real.

Rather than jump between two solutions based upon the two different types of poles, we can switch to a set of alternate parameters, $A_{n}, \delta_{n}$, and $\Gamma_{n}^{2}$, which are all real and will preserve their relationships with the poles in a meaningful way. The ultimate goal is to achieve a single mathematical model which will transition smoothly from the narrowband Lorentz (NBL), which uses poles depicted in Fig. 1(a), to the wideband Lorentz (WBL), which uses poles depicted in Fig. 1(b), through just these parameters.

Replacing the numerators in (8) with $-A_{n}$ simply helps with compactness. The reason for the negative sign in front of $A_{n}$ is that each pole pair in this simplest of groupings must represent an absorbing transition, and it is usually preferable that parameters be positive, whenever possible. Of the three, $A_{n}, \delta_{n}$, and $\Gamma_{n}^{2}$, it turns out that only the last parameter can be $\leqslant 0$.

As shown in Fig. 1, one thing shared by both types of pole pairs is that their respective midpoints must lie on the negative imaginary axis, below the origin by a distance which shall be defined as $\delta_{n}$. With the possible exception of a simple pole at the origin, all poles must be below the real axis in the $\omega$ plane 
for physical stability. Because of this, all midpoint distances $\delta_{n}$ must be real and greater than zero. We can also define a scalar, $\Gamma_{n}$, which when added to or subtracted from the midpoint, will arrive precisely onto one of the poles. Note that $\Gamma_{n}$ can be purely real as in Fig. 1(a) or purely imaginary as in Fig. 1(b); either way this means that $\Gamma_{n}^{2}$ is guaranteed to be real. The mathematical definitions are

$$
\delta_{n} \equiv \frac{P_{1, n}+P_{2, n}}{-2 i}
$$

and

$$
\Gamma_{n}^{2} \equiv-P_{1, n} P_{2, n}-\delta_{n}^{2}
$$

With the definitions established for $A_{n}, \delta_{n}$, and $\Gamma_{n}^{2}$, (8) can now be written in its unified Lorentzian form:

$$
\chi(\omega)=\sum_{n} \frac{A_{n}}{\left(\delta_{n}^{2}+\Gamma_{n}^{2}\right)-2 i \delta_{n} \omega-\omega^{2}},
$$

with the restrictions that

$A_{n}>0$ for each pole pair which absorbs energy;

$\delta_{n}>0$ for physical stability; and

$\Gamma_{n}^{2} \geqslant-\delta_{n}^{2}$ for if $\Gamma_{n}^{2}$ were allowed to be less than $-\delta_{n}^{2}$, one of the poles would be forced into the upper $\omega$ half plane.

\section{Time-domain kernels}

In the time domain, Lorentzian susceptibility is often represented by sine functions, whose amplitudes are diminishing exponentially in time, such as the examples appearing in [10] and [17], but if $\Gamma_{n}^{2}$ ever equals zero, which in fact it does for the modified Debye model, the denominator in (15) can cause a computational error.

$$
\chi(t)=u(t) \sum_{n} \frac{A_{n}}{\sqrt{\Gamma_{n}^{2}}} \exp \left(-\delta_{n} t\right) \sin \left(\sqrt{\Gamma_{n}^{2}} t\right) .
$$

For the time-domain kernels, it may be preferable to use extended sinc function representations,

$$
\chi(t)=u(t) \sum_{n} A_{n} t \exp \left(-\delta_{n} t\right) \operatorname{sinc}\left(\sqrt{\Gamma_{n}^{2} t^{2}}\right),
$$

where

$$
\operatorname{sinc}\left(x^{1 / 2}\right)=1-\frac{x}{3 !}+\frac{x^{2}}{5 !}-\frac{x^{3}}{7 !}+\cdots,
$$

which is true for any real value $x=\Gamma_{n}^{2} t^{2}$. The sinc functions go smoothly from negative values of $\Gamma_{n}^{2}$ (for the WB Lorentzians) to positive values (for the NB Lorentzians), and they have no problems at the particular cases when $\Gamma_{n}^{2}$ equals $-\delta_{n}^{2}$ (the Drude case) or when $\Gamma_{n}^{2}$ equals zero (the modified Debye, a.k.a. "limiting" case, "borderline" case, or "critically damped" case). Note how elegantly separated the three parameters are in (16); all the functions are real and all the parameters are real, keeping in mind that we only need to work with $\Gamma_{n}^{2}$ and never directly with $\Gamma_{n}$.

\section{E. Proof that duplicate poles are allowed}

The proof starts with the rational function as expressed in (1) and examines the consequences if in the denominator a pair of identical poles exists. If we assign each twin the generally complex value of $P_{k}$, this would imply a partial fraction decomposition contributor, not originally included on the right side of (6) in the form of

$$
\chi_{k}(\omega)=\frac{B_{k}}{\left(\omega-P_{k}\right)}+\frac{C_{k}}{\left(\omega-P_{k}\right)^{2}} .
$$

In the time domain the contribution is given by

$$
\chi_{k}(t)=-u(t)\left(i B_{k}+C_{k} t\right) \exp \left(-i P_{k} t\right) .
$$

To be physically realizable, the contribution at $t=0^{+}$must vanish; therefore, $B_{k}$ must be zero. This leaves

$$
\chi_{k}(\omega)=\frac{C_{k}}{P_{k}^{2}-2 P_{k} \omega+\omega^{2}} .
$$

Causal-based symmetry requires $P_{k}$ in the denominator to be purely imaginary, not generally complex. From this we can infer that all complex poles with a nonzero real component must be simple.

Causality forces $C_{k}$ to be real, and physical realizability requires it to be less than zero. Since there is no analytical or physical reason that $C_{k}$ must be equal to zero, we must conclude that twin poles are indeed allowed, but only the purely imaginary poles on the negative imaginary $\omega$ axis.

Twin poles at the origin are not allowed, however, because if $P_{k}=0$ were permitted in (20), this would imply the presence of a purely lossless susceptibility contributor which $\rightarrow(-\infty)$ as $\omega \rightarrow 0$; both implications are physically unrealizable.

The main thrust of the above proof is this; by examining (20) we can see that even if some of the poles were not simple, for their susceptibility we would still arrive at an equivalent construct as the one which appears in (14).

\section{F. Role of the Debye model}

Strictly speaking, the Debye model for susceptibility does not exist in this system; there is no combination of realizable parameters in (14) which will reduce it to a Debye dispersion model. This does not mean the Debye is not a useful model. It is accurate when the frequency of interest is sufficiently small that the $\omega^{2}$ term in the denominator in (14) is negligible. This occurs quite frequently in the rf spectral region but nowhere else. To understand this we need to look at an alternative form for (14), one which is based on the quality factors, $Q_{n}$. Such an equation might look like this:

$$
\chi(\omega)=\sum_{n} \frac{\left(A_{n} / 4 \delta_{n}^{2}\right)}{Q_{n}^{2}-i \frac{\omega}{2 \delta_{n}}-\left(\frac{\omega}{2 \delta_{n}}\right)^{2}},
$$

where the full width half maximum (FWHM) quality factors $Q_{n}$ are defined by

$$
Q_{n} \equiv \frac{\sqrt{\delta_{n}^{2}+\Gamma_{n}^{2}}}{2 \delta_{n}} .
$$

Note that the magnitude of the linear coefficient in the denominator of (21) is precisely equal to the square root of 


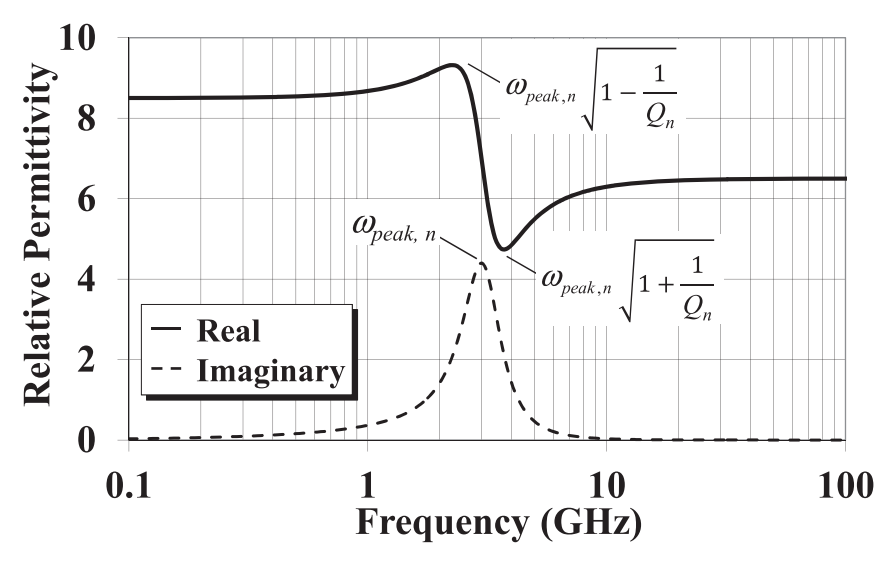

FIG. 2. Complex components of a classic narrowband Lorentzian dispersion susceptibility model near one excitation.

the quadratic term's magnitude. In this form then, the relative significance of the quadratic term to the linear term is exactly equal to the linear term's magnitude.

Generally we are only interested in making measurements in the frequency ranges around the resonance or relaxation peak of the imaginary component of the susceptibility, since in the wings the contributions are small or nearly constant. Let $\omega_{\text {peak }, n}$ be defined as the frequency value when the imaginary component of the $n$th transition's susceptibility contribution is at its peak (see Fig. 2). Solving (21) for the maximum imaginary component of the susceptibility yields

$$
\frac{\omega_{\text {peak }, n}}{2 \delta_{n}}=\sqrt{\frac{\sqrt{\left(1-2 Q_{n}^{2}\right)^{2}+12 Q_{n}^{4}}-\left(1-2 Q_{n}^{2}\right)}{6} .}
$$

A plot of this ratio as a function of the $Q$ factor appears in Fig. 3. It reveals that the relative significance of the quadratic term is at least one order of magnitude less than that of the linear term whenever the $Q$ factor is $\leqslant 0.3$. In fact the significance is nearly equal to $Q^{2}$ for any $Q \leqslant 0.5$.

$$
\frac{\omega_{\text {peak }, n}}{2 \delta_{n}} \approx Q_{n}^{2} \text { for } Q_{n} \leqslant 0.5 \text {. }
$$

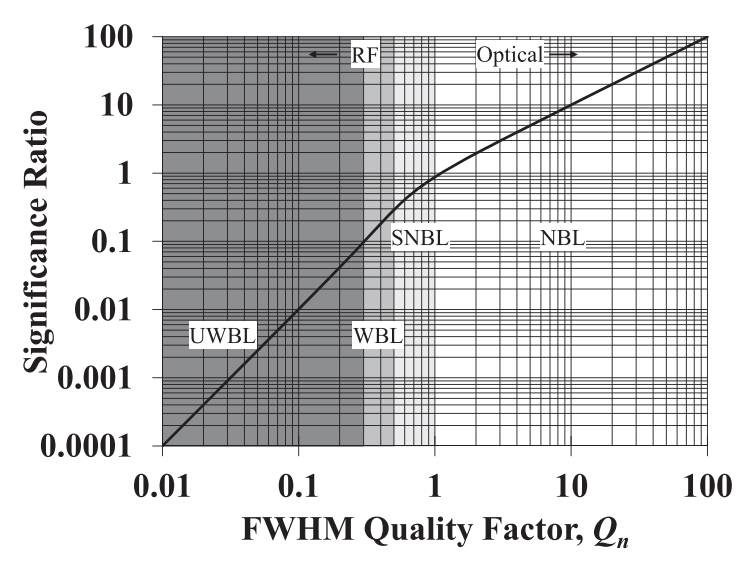

FIG. 3. Significance of the quadratic term relative to the linear term as a function of quality factor $Q_{n}$ at the peak frequency $\omega_{\text {peak }, n}$.
For any $Q$ factor greater than 1 , the ratio of the magnitude of the quadratic coefficient to that of the linear one is approximately equal to $Q$. So in the denominator, not only is the quadratic term non-negligible, it dominates. In the optical region the $Q$ factors are typically, if not always observed to be, greater than 1, and as such, Debye-like susceptibility behavior would be mathematically forbidden.

$$
\frac{\omega_{\text {peak }, n}}{2 \delta_{n}} \approx Q_{n} \text { for } Q_{n} \geqslant 1 .
$$

\section{G. Four classes of Lorentzian behavior}

Although some researchers such as in [18] refer to only two classes of Lorentzian behavior regions, the wideband $(Q$ $\leqslant 0.5)$ and the narrowband $(Q>0.5)$, these distinctions are made purely on their behavior in the time domain. ${ }^{1}$ However, if we include behavior in the frequency domain, then four regions (which are depicted in Fig. 3) of distinctly different behaviors exist for the Lorentzian model; they are all described along with their corresponding $Q$ ranges in Table I.

In the frequency domain, the classic NBL susceptibility such as the one shown in Fig. 2 exhibits two spikes, one maximum, and one minimum. But as also revealed in the figure, the maximum only occurs for $Q \geqslant 1$. Since in the small region, $0.5<Q<1$, the Lorentzian still oscillates in the time domain but only has one of the two typical NBL spikes in the frequency domain; this investigator refers to this region of unique behavior as seminarrowband Lorentzian (SNBL).

When $Q$ is less than or equal to 0.5 , there is no oscillation in the time domain, but there is always a minimum in the frequency domain. This behavior is referred to as typical wideband Lorentzian (WBL) behavior in the time domain. However, as discussed in the previous section, when $Q$ is less than about 0.3 , the susceptibility term for $\omega \leqslant \omega_{\text {peak }, n}$ does not behave as a classical wideband Lorentzian; the quadratic term in the denominator of (21) becomes negligible, and the one frequency-domain minimum migrates inversely as $Q^{2}$ towards higher frequencies away from $\omega_{\text {peak }, n}$, oftentimes by several orders of magnitude. At frequencies lower than or equal to the peak frequency the behavior becomes very Debye-like. Understanding that this behavior is not really Debye, nor is it wideband Lorentzian, this investigator chooses to call this behavior ultrawideband Lorentzian (UWBL). The electric susceptibility may behave like a Debye material near $\omega_{\text {peak }, n}$ and below, but extrapolating it to higher frequencies should be avoided because at some point, the quadratic term ceases to be negligible. In summary, there is no Debye susceptibility in the complete model, just ultrawideband Lorentzian in its place.

\section{DATA FITTING}

\section{A. Compact formula}

Even with the various Lorentzian susceptibility equations (8), (14), and (21), fitting them to data can be frustrating due to the significant number of unknowns. Traditionally the investigator will extract as few terms from the summation

\footnotetext{
${ }^{1}$ The $Q$ factors used by the researchers in [18] have been converted from their half width half maximum (HWHM) convention values.
} 
TABLE I. Lorentzian characteristics and classes.

\begin{tabular}{|c|c|c|c|c|}
\hline$Q$ & Freq. domain & Time domain & Lorentz class & Comment \\
\hline $1 \leqslant Q<\infty$ & Two real extrema & Oscillation & Narrowband & Double spikes \\
\hline $0.5<Q<1$ & One real extremum & Oscillation & Seminarrowband & Single spike \\
\hline $0.3<Q \leqslant 0.5$ & One real extremum & No oscillation & Wideband & Mod Debye at $Q=0.5$ \\
\hline $0<Q \leqslant 0.3$ & One real extremum & No oscillation & Ultrawideband & Debye-like \\
\hline$Q=0$ & $\begin{array}{c}\chi^{\prime \prime} \rightarrow \infty \\
\text { as } \omega \rightarrow 0\end{array}$ & No oscillation & Drude & Dc conductivity \\
\hline
\end{tabular}

as possible and then combine all the other terms together into one "constant" and call it $\chi_{\infty}, \varepsilon_{\infty}$, or $\mu_{\infty}$. One of the unknowns includes how many terms must be extracted. In the rf region the peaks tend to be well isolated from each other so commonly only one Lorentzian term is needed, at times two. In this section, methods shall be revealed which have been found, through experimentation, to be quite successful in this region.

A $Q$ factor of zero (the Drude case) corresponds to when one of the poles is at the origin, and it deserves special consideration. Since this pole appears at most only once in the rational function expressed in (1), only one pole pair can have a $Q$ factor of zero. We will assign this specific case to $n=0$ and extract it from the summation in (14).

$$
\chi_{0}(\omega)=\frac{i \sigma_{0}}{\omega-i \omega^{2} / 2 \delta_{0}}=\frac{i \sigma_{0}}{\omega}-\frac{\sigma_{0} \tau_{0}}{1-i \omega \tau_{0}},
$$

where $\tau_{0}=1 / 2 \delta_{0}$. We can do this because $\delta_{0}$ can never be zero.

Since none of the remaining $\left(\Gamma_{n}^{2}+\delta_{n}^{2}\right)$ values in the summation in (14) can be zero, we are free to divide by them, resulting in

$$
\chi(\omega)=\frac{i \sigma_{0}}{\omega}-\frac{\sigma_{0} \tau_{0}}{1-i \omega \tau_{0}}+\sum_{n=1} \frac{\Delta_{n}}{1-i \omega \tau_{n}-\omega^{2} T_{n}^{2}},
$$

where

$T_{n}^{2}=1 /\left(\delta_{n}^{2}+\Gamma_{n}^{2}\right), \Delta_{n}=A_{n} T_{n}^{2}$, and $\tau_{n}=2 \delta_{n} T_{n}^{2}$ for $n>0$.

Note that the second term in (27) is a pure Debye term; it is necessary for physical realizability at high frequency, but for compactness, it can be incorporated back into the summation, for example, as

$$
\chi(\omega)=\frac{i \sigma_{0}}{\omega}+\sum_{n=0} \frac{\Delta_{n}}{1-i \omega \tau_{n}-\omega^{2} T_{n}^{2}},
$$

where we let $T_{0}^{2}=0$, and $\Delta_{0}=-\sigma_{0} \tau_{0}$ (the only dual-pole case where $\Delta \leqslant 0$ ) for $n=0$.

At some point all optimization algorithms start refining the parameters by increasingly smaller increments. By working with parameters which represent periods of time, such as $\tau_{n}$ and $T_{n}^{2}$, it has been found that the algorithms tend to converge faster in the frequency domain than if they were working on solving for their multiplicative inverses.

For a single Lorentzian response, the compact version of (28) becomes

$$
\chi(\omega)=\chi_{\infty}+\frac{i \sigma_{0}}{\omega}+\frac{\Delta_{1}}{1-i \omega \tau_{1}-\omega^{2} T_{1}^{2}},
$$

where

$$
\chi_{\infty} \equiv \sum_{n \neq 1} \frac{\Delta_{n}}{1-i \omega \tau_{n}-\omega^{2} T_{n}^{2}}
$$

As is often the case in rf, in order to accurately extract values for the unknown parameters, we have to assume that the input data are from a neighborhood that is isolated sufficiently from the other peaks so that $\chi_{\infty}$ is well approximated by a real constant, but sometimes in cases needing higher precision this assumption may be insufficient. Caution is warranted.

For most dielectric materials there is no dc conductivity term; therefore, we often have the benefit of being able to fit susceptibility in the rf region using an inverted form of (29).

$$
\frac{1-i \omega \tau_{1}-\omega^{2} T_{1}^{2}}{\Delta_{1}}=\frac{1}{\chi(\omega)-\chi_{\infty}} .
$$

Note that (31) could be fit using a simple linear leastsquares-fit routine if only we had a way of estimating $\chi_{\infty}$.

\section{B. Estimating $\chi_{\infty}$}

If we extract the imaginary part of (31) we get

$$
\frac{\omega \tau_{1}}{\Delta_{1}}=\frac{\chi^{\prime \prime}(\omega)}{\left[\chi^{\prime}(\omega)-\chi_{\infty}\right]^{2}+\chi^{\prime \prime 2}(\omega)},
$$

where $\chi^{\prime}(\omega)$ and $\chi^{\prime \prime}(\omega)$ are, respectively, the real and imaginary components of the susceptibility.

With some rearrangement, and the following substitutions, $x=\chi^{\prime}(\omega)-\chi_{\infty}, y=\chi^{\prime \prime}(\omega)$, and $z=1 / \omega$, we arrive at an equation which is recognizable from analytic geometry as describing a tilted elliptic cone surface (see Fig. 4),

$$
x^{2}+y^{2}=a y z,
$$

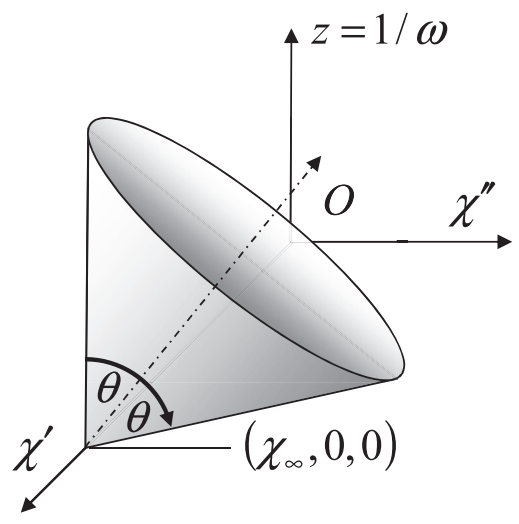

FIG. 4. Tilted elliptical cone surface. 
where $a=\tan (2 \theta)=\Delta_{1} / \tau_{1}$ and $\chi_{\infty}$ is the apex of the cone.

We can perform a least-squares fit on (33), minimizing the sum of the square of the differences between $x^{2}+y^{2}$ and ayz with respect to $a$ and $\chi_{\infty}$. The two conditions for minimum are

$$
a=\sum S_{k} t_{k} / \sum t_{k}^{2}
$$

and

$$
a=\sum S_{k} x_{k} / \sum x_{k} t_{k}
$$

where $S_{k} \equiv x_{k}^{2}+y_{k}^{2}, t_{k} \equiv y_{k} z_{k}$, and index $k$ refers to each data point. Eliminating $a$ by combining (34) and (35) we arrive at

$$
\sum S_{k} x_{k} \sum t_{k}^{2}-\sum S_{k} t_{k} \sum x_{k} t_{k}=0 .
$$

Equation (36) is a cubic equation with respect to $\chi_{\infty}$ and an attractive attribute of cubic equations is that they are guaranteed to have either one or three real solutions. So from this one LSF we have at least one, and possibly three sets of estimates for $\chi_{\infty}$ and the ratio $a$. Another noteworthy fact is that (36) is derived without any reference to $\Gamma_{1}^{2}$ (through $T_{1}^{2}$ ); this means the equation should apply equally to any Lorentzian regardless of $Q$.

\section{Estimating the remaining Lorentzian parameters}

If we extract the real part of (31) we get

$$
\frac{1-\omega^{2} T_{1}^{2}}{\Delta_{1}}=\frac{\left[\chi^{\prime}(\omega)-\chi_{\infty}\right]}{\left[\chi^{\prime}(\omega)-\chi_{\infty}\right]^{2}+\chi^{\prime \prime 2}(\omega)} .
$$

The motivation here is to derive a linear equation we can fit to give us $T_{1}^{2}$, and either $\tau_{1}$ or $\Delta_{1}$. Many algebraic manipulations are available but many of them fail to converge.

By combining (32) and (37) we can reduce the order with respect to $\omega$ in order to reduce sensitivity and improve stability.

$$
\begin{aligned}
& {\left[\chi^{\prime}(\omega)-\chi_{\infty}\right]^{2}+\chi^{\prime \prime 2}(\omega)-\frac{\Delta_{1} \chi^{\prime \prime}(\omega)}{\omega \tau_{1}} \omega^{2} T_{1}^{2}} \\
& \quad=\Delta_{1}\left[\chi^{\prime}(\omega)-\chi_{\infty}\right] .
\end{aligned}
$$

By introducing a new constant, the low frequency constant $\chi_{\mathrm{dc}} \equiv \chi(\omega=0)$ and realizing that $\Delta_{1}$ equals $\chi_{\mathrm{dc}}-\chi_{\infty}$, and with some rearrangement we arrive at

$$
\begin{aligned}
& \chi^{\prime 2}(\omega)+\chi^{\prime \prime 2}(\omega)-\left(\chi_{\mathrm{dc}}+\chi_{\infty}\right) \chi^{\prime}(\omega)+\chi_{\mathrm{dc}} \chi_{\infty} \\
& \quad=a T_{1}^{2} \omega \chi^{\prime \prime}(\omega) .
\end{aligned}
$$

Using a linear least-squares fit, the constants $\chi_{\mathrm{dc}}$ and $T_{1}^{2}$ can be found, and from those constants, so can $\Delta_{1}$ and $\tau_{1}$.

Traditionally at this point of the analysis it is customary to conduct a root polishing procedure, such as a LevenbergMarquardt process [19], on the constants to minimize rms error from (29); however, one must keep in mind that the minimum rms is not always the correct answer, especially if it leads to nonphysical results.

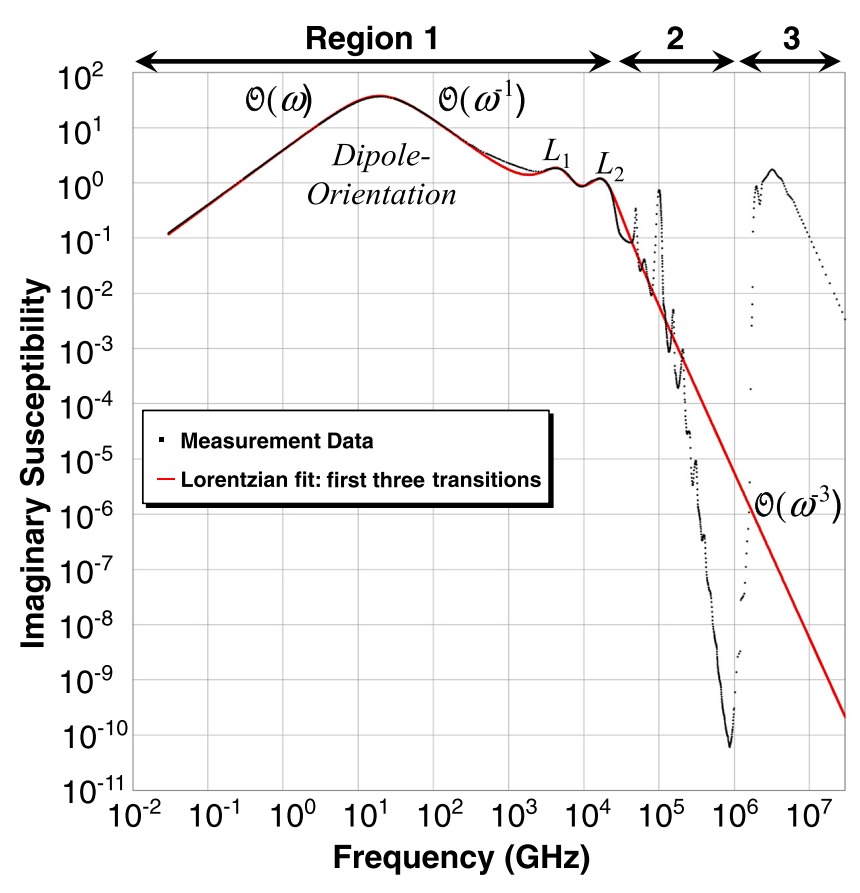

FIG. 5. Imaginary component of permittivity of liquid water at $25{ }^{\circ} \mathrm{C}$; data dots from 550 to $1200 \mathrm{THz}$ were measured values from Mason et al. [20]; all other data dots were compiled by Segelstein [9]. Lorentzian fit of the first three transitions is shown as a smooth line.

\section{MODELING THE PERMITTIVITY OF LIQUID WATER}

\section{A. Problem}

Water has a rich and mysterious spectrum, confounding experts to model and explain for close to 200 years. In 2007 Shubitidze and Osterberg [7] reduced the number of spectral regions to three; region 1 consisting of purely rotational or orientational transitions; region 2, vibrational transitions; and region 3, vibronic transitions which at present can only be explained through quantum mechanics; this is outside the scope of this paper.

In Fig. 5 appear the data from Segelstein's compilation [9] which was used by Shubitidze and Osterberg [7]; however, to make the fit in the present model even more challenging, the plot has been updated by substituting the latest measurements conducted in 2016 by Mason et al. [20] in the spectral range of $550-1200 \mathrm{THz}$, which revealed that a new absorption minimum at $871.5 \mathrm{THz}(344 \mathrm{~nm})$ exists which is 15 times lower than the one reported by Segelstein at $631.1 \mathrm{THz}$ $(475 \mathrm{~nm})$. The measured data collected by Segelstein were based largely on absorption measurements, with most of the real values coming from Kramers-Kronig calculations. Because they tend to be more accurate, only the imaginary components of the data were fit to (28) keeping in mind that the Drude contribution must have a minimum of two poles to be rigorously physically realizable (RPR). The second pole can become especially significant when dealing with water found in living tissue where the water is highly conductive due to the significant concentration of electrolytes.

We will begin our fitting by focusing solely on the three transitions in region 1 . In the traditional approach previous 
investigators such as Segelstein [9] assumed that these transitions were of Debye type and that water was "nonconductive" and therefore, ignored the dc conductivity. The latter assumption appears justified since a quick calculation reveals that at the low conductivity value of $5.501 \times 10^{-6} \mathrm{~S} / \mathrm{m}$ (water's conductivity at $25^{\circ} \mathrm{C}$ according to ASTM Standard D1125-95 2005) the significance of the dc conductivity pole contribution would be a barely perceptible $3 \%$ at the lowest frequency of $30 \mathrm{MHz}$ and would decline rapidly as $\omega^{-2}$.

To illustrate the problem better from the historical vantage we will also assign water a dc conductivity of zero; however, since single-pole transitions are forbidden in the complete model, we will insist on fitting a dual-pole (complex Lorentzian) function to each of the three significant transitions occurring in region 1; the result is shown in Fig. 5. A problem appears immediately in the vicinity of the steep decline at $30 \mathrm{THz}$ for the $L_{2}$ libration transition, a place where in 1973 Hasted [21] could not get a good fit (nor had anyone else for that matter); he referred to this as "a known anomaly in the Debye theory." Most profound though is that the high frequency wing of the traditional fit cuts right across the optical valley. The simple truth is that the dual-pole models, for which the imaginary susceptibility approaches 0 at higher frequencies as $\omega^{-3}$, are insufficient to account for the rapid drop in absorption observed in the measured data; therefore, we must admit there is a need to introduce a refinement to the traditional model to account for this, and since the model expressed in (2) is complete, that refinement must come in the form of more poles.

\section{B. Solution}

After giving it some thought, this investigator is drawn to the conclusion that the simplest refinement must come in the form of a dual-pole reemission term which returns a portion of the absorbed energy. The simplest of refinements should be something we can add (or subtract) or ignore at our discretion; it should therefore be independent; that is, its parameters should not force us to change other parameters in the material system, even though we may freely choose to. Obviously, a reemitter must also be physically realizable, because the addition of a single unrealizable contributor to a realizable system always makes the result unrealizable. This is why we cannot choose a single-pole refinement term; at a minimum a reemitter requires two poles, and because we require physical independence, it must be in the form of a Lorentzian.

The model for susceptibility has now become a summation of transition representations, each described by the difference between two Lorentzians. This is permissible as long as the imaginary component of the difference is greater than zero for all real frequencies; obviously, a transition's reemitter cannot emit more energy than it absorbs. By modifying (28) to include the reemitters we arrive at (40), a general susceptibility model for materials using a quadruple-pole representation.

Naturally, in order to carry out the least-squares fit, it is better to use a form which avoids subtractive cancellation as much as possible. Such a form appears in (41). When conducting the fit the user basically focuses on adjusting the first Lorentzian and the delicate difference between it and the second Lorentzian expression rather than on the Lorentzians

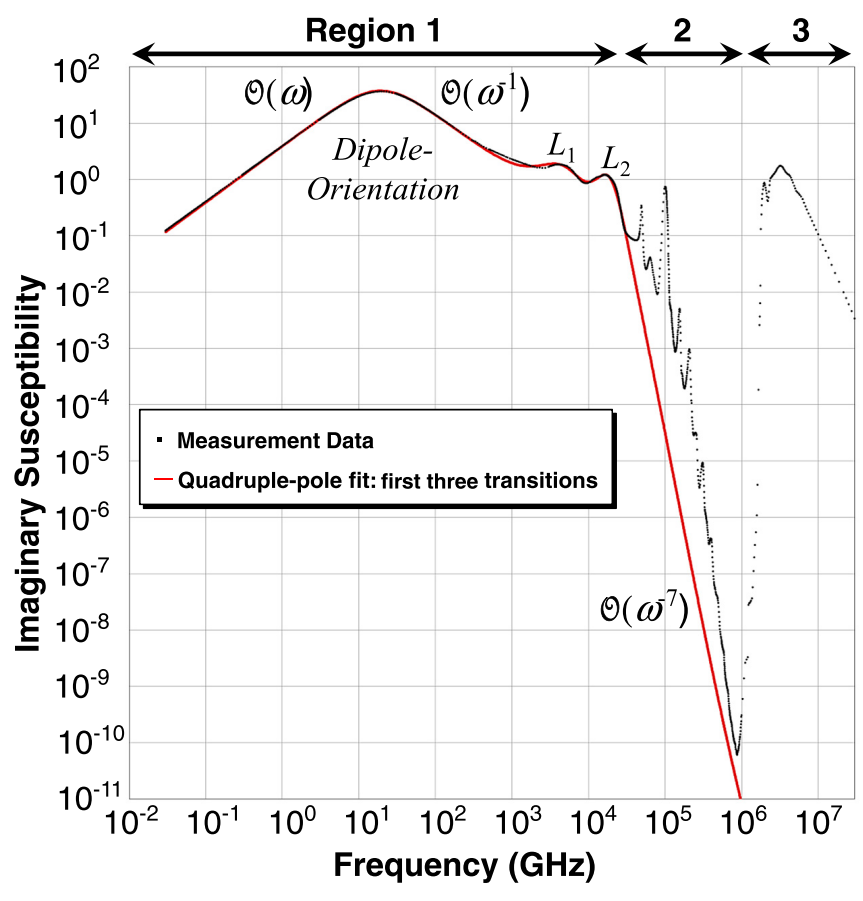

FIG. 6. Imaginary component of permittivity of liquid water at $25^{\circ} \mathrm{C}$; data dots from 550 to $1200 \mathrm{THz}$ were measured values from Mason et al. [20]; all other data dots were compiled by Segelstein [9]. The quadruple-pole fit for the first three transitions is shown as a smooth line.

themselves. Equation (41) is a truly unified model; it can represent Drude, Lorentzian, and the quadruple-pole transitions. It can even represent Debye transitions. Note that if $\Delta_{2, n}=0$ (which it does for almost all materials), the rational expression under the summation reverts to a single Lorentzian; further, if we let $T_{n}^{2}=0$, the expression then becomes a pure Debye. Of course under the current theory it is understood that when we make such an assignment we are acknowledging that while $T_{n}^{2}$ is not formally allowed to equal zero, in the band of interest it is well approximated by zero.

The result of using the model in (41) is a much improved fit, shown in Fig. 6, especially in the steep decline at $30 \mathrm{THz}$ which gave Hasted [21] such difficulty. Another interesting fact is that even when the fitting program was experimentally forced, it could not fit the rf transition at $19 \mathrm{GHz}$ as a Debye; to get below the absorption minimum requires a quality factor of around $0.05 \pm 0.03$ which is more than small enough to make the Lorentzian measurably indistinguishable from a Debye model in the rf region. What is most important though is that the model is able to achieve the $\omega^{-7}$ slope necessary to get under the optical valley, a slope which cannot be achieved with fewer than four poles.

$$
\begin{gathered}
\chi(\omega)=\frac{i \sigma_{0}}{\omega}+\sum_{n=0}\left(\frac{\Delta_{1, n}}{1-i \omega \tau_{1, n}-\omega^{2} T_{1, n}^{2}}\right. \\
\left.-\frac{\Delta_{2, n}}{1-i \omega \tau_{2, n}-\omega^{2} T_{2, n}^{2}}\right), \\
\chi(\omega)=\frac{i \sigma_{0}}{\omega}+\sum_{n=0} \frac{\left(\Delta_{n}+\Delta_{2, n}\right) B_{n}(\omega)}{A_{n}(\omega)\left[B_{n}(\omega)+\Delta_{2, n} A_{n}(\omega)\right]},
\end{gathered}
$$




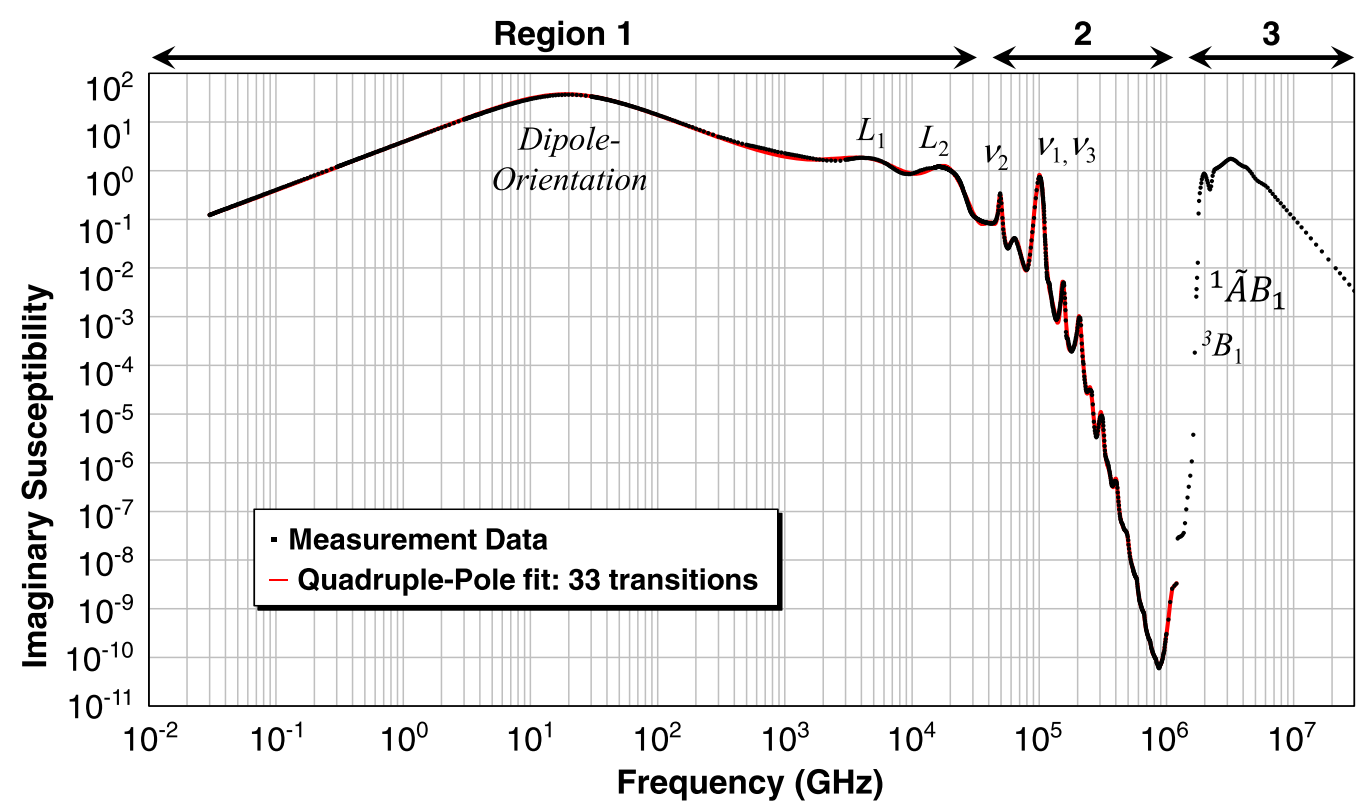

FIG. 7. Imaginary component of permittivity of liquid water at $25^{\circ} \mathrm{C}$; data dots from 550 to $1200 \mathrm{THz}$ were measured values from Mason et al. [20]; all other data dots were compiled by Segelstein [9]. The quadruple-pole fit for 33 transitions is shown as a smooth line.

where $\Delta_{n} \equiv \Delta_{1, n}-\Delta_{2, n}, \quad \tau_{n} \equiv \tau_{1, n}, T_{n}^{2} \equiv T_{1, n}^{2}, \quad c_{n}(\omega) \equiv$ $\Delta_{1, n} \tau_{2, n}-\Delta_{2, n} \tau_{1, n}, \quad d_{n}(\omega) \equiv \Delta_{1, n} T_{2, n}^{2}-\Delta_{2, n} T_{1, n}^{2}$, $A_{n}(\omega)=1-i \omega \tau_{n}-\omega^{2} T_{n}^{2}$, and $B_{n}(\omega)=\Delta_{n}-i \omega c_{n}-\omega^{2} d_{n}$.

Based on this success, we will now bring the vibrational transitions occurring in region 2 into our model. From the combined data collection by Segelstein [9] and the pure water measurements by Mason et al. [20], on close inspection there appear to be 33 peaks and shoulders between $19 \mathrm{GHz}$ and $1200 \mathrm{THz}$, most of which correspond to the 14 overtones of $v_{3}$ and combinations of those overtones with a single quantum of $v_{2}$. Since we are striving to be more rigorous and complete, we will now include the dc conductivity in the fit. The results shown in Fig. 7 are in a word, excellent, with an rms error of $4.2 \%$ on the log scale. The parameter values can be found in Table II. Amazingly, the parameters for the whole spectrum model easily fit on one page.

To complete the test, the resultant real component for the permittivity is plotted in Fig. 8. A strong advantage of

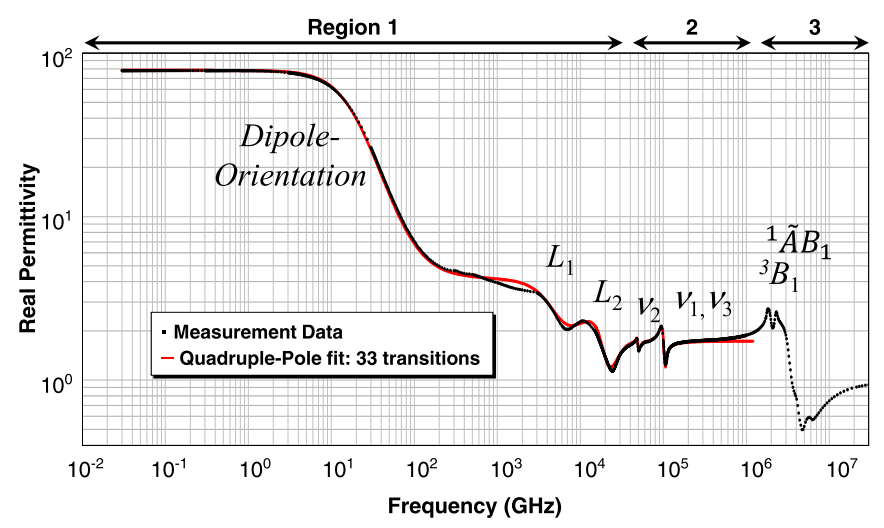

FIG. 8. Real component of permittivity of liquid water at $25^{\circ} \mathrm{C}$; data dots from 550 to $1200 \mathrm{THz}$ were measured values from Mason et al. [20]; all other data dots were compiled by Segelstein [9]. The quadruple-pole fit for 33 transitions is shown as a smooth line. an analytic model is that the real component is obtained automatically from the same parameters as the imaginary one. The sole difference is that the real permittivity has the $\varepsilon_{\infty}$ parameter, which was found to be $1.73 \pm 0.02$.

The fit is good, but there appears to be significant disagreement with the data around $1500 \mathrm{GHz}$. It bears stating that most of the data dots which appear in the figures are simply modeled from measurements and do not represent the actual measurements which often appear quite scattered. After further investigation, it was discovered that the data in this region are based on measurements performed by Afsar and Hasted [22] which were conducted at $19{ }^{\circ} \mathrm{C}$, not $25^{\circ} \mathrm{C}$, as the data points surrounding them. This would easily account for both the magnitude and the direction of the discrepancy.

During the development of the mathematical model, any attempt to conjure a physical process with which to model the electric susceptibility of water was deliberately avoided in order to take full advantage of the power of network theory. So long as we rigorously adhere to a few simple rules, the outcome is guaranteed to be physically realizable, even though we may not know its physical form. Only after deriving (40) was the exercise ever attempted.

Figure 9 shows an electric circuit which can be used to study Lorentzian behavior; it is a simple assembly of $L R C$ (inductance, resistance, and capacitance) electronic elements

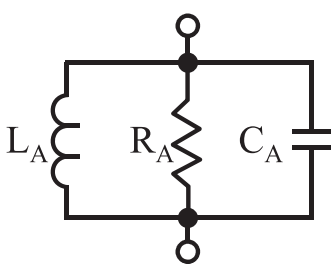

$$
Z_{\mathrm{A}}=\left[\frac{i}{\omega L_{\mathrm{A}}}+R_{\mathrm{A}}^{-1}-i \omega C_{\mathrm{A}}\right]^{-1}
$$

FIG. 9. A circuit analog for modeling a complex Lorentzian response. 
TABLE II. Lorentzian parameters for modeling permittivity of liquid water at $25^{\circ} \mathrm{C}$. Note: numerals in square brackets indicate the power of 10 .

\begin{tabular}{|c|c|c|c|c|c|c|c|c|}
\hline$n$ & $\begin{array}{l}\text { Peak } \\
(\mathrm{GHz})\end{array}$ & $\begin{array}{l}\text { Assignment } \\
\left(v_{1}, v_{2}, v_{3}\right)\end{array}$ & $\Delta_{n}$ & $\begin{array}{c}\tau_{n} \\
\text { (ns/cycle) }\end{array}$ & $\begin{array}{c}T_{n}^{2} \\
\text { (ns/cycle) })^{2}\end{array}$ & $\Delta_{2, n}$ & $\begin{array}{c}c_{n} \\
\text { (ns/cycle) }\end{array}$ & $\begin{array}{c}d_{n} \\
(\mathrm{~ns} / \text { cycle })^{2}\end{array}$ \\
\hline 0 & $2.7223[+04]$ & Drude pair & $-6.61[-09]$ & $3.67[-05]$ & 0 & 0 & 0 & 0 \\
\hline 1 & $1.9089[+01]$ & Dipole & $7.393[+01]$ & $5.250748[-02]$ & $6.372140[-06]$ & $1.773454[-01]$ & $1.562453[-02]$ & $7.192095[-07]$ \\
\hline 2 & $3.9617[+03]$ & $\mathrm{L}_{1}$ & $2.0009[+00]$ & $2.787242[-04]$ & $3.366230[-08]$ & $3.855433[-02]$ & $1.277124[-04]$ & $3.360751[-09]$ \\
\hline 3 & $1.7709[+04]$ & $\mathrm{L}_{2}$ & $7.3227[-01]$ & $3.8839971[-05]$ & $2.7703948[-09]$ & $3.3395439[-01]$ & $3.3058241[-05]$ & $8.1767155[-10]$ \\
\hline 4 & $4.1668[+04]$ & $2 \mathrm{~L}_{2}$ & $2.01455[-02]$ & $1.0471383[-05]$ & $5.4748638[-10]$ & $6.844104[-02]$ & $3.6874461[-07]$ & $8.6429546[-12]$ \\
\hline 5 & $4.9268[+04]$ & $0,1,0$ & $1.3249[-02]$ & $1.869905[-06]$ & $4.110942[-10]$ & $1.30666[-01]$ & $5.155956[-08]$ & $5.396545[-12]$ \\
\hline 6 & $6.4376[+04]$ & $0,1,0+\mathrm{L}_{2}$ & $6.7667[-03]$ & $4.925086[-06]$ & $2.3511621[-10]$ & $5.67074[-02]$ & $6.259506[-08]$ & $1.4186308[-12]$ \\
\hline 7 & $9.5313[+04]$ & $0,2,0$ & $1.61849[-02]$ & $1.1594759[-06]$ & $1.0974029[-10]$ & $1.583745[-01]$ & $3.885802[-08]$ & $1.7507161[-12]$ \\
\hline 8 & $1.0025[+05]$ & $1,0,0$ & $2.45812[-02]$ & $5.437274[-07]$ & $9.942913[-11]$ & $2.424527[-02]$ & $3.9791716[-08]$ & $2.4217991[-12]$ \\
\hline 9 & $1.0477[+05]$ & $0,0,1$ & $1.42076[-02]$ & $4.8348621[-07]$ & $9.104601[-11]$ & $1.403999[-02]$ & $2.0472389[-08]$ & $1.2833647[-12]$ \\
\hline 10 & $1.2316[+05]$ & $0,0,1+\mathrm{L}_{2}$ & $1.9118[-04]$ & $1.551646[-06]$ & $6.53246[-11]$ & $1.60969[-04]$ & $8.10805[-10]$ & $1.115324[-14]$ \\
\hline 11 & $1.5291[+05]$ & $0,1,1$ & $2.39856[-04]$ & $4.551342[-07]$ & $4.271926[-11]$ & $2.344489[-04]$ & $3.233935[-10]$ & $1.0092290[-14]$ \\
\hline 12 & $1.8291[+05]$ & $0,1,1+\mathrm{L}_{2}$ & $1.9471[-05]$ & $9.4208[-07]$ & $2.96655[-11]$ & $1.68012[-05]$ & $5.07980[-11]$ & $5.24268[-16]$ \\
\hline 13 & $2.0574[+05]$ & $0,0,2$ & $5.2646[-05]$ & $3.86079[-07]$ & $2.35863[-11]$ & $5.09824[-05]$ & $5.99036[-11]$ & $1.215592[-15]$ \\
\hline 14 & $2.5047[+05]$ & $0,1,2$ & $2.0265[-06]$ & $3.86891[-07]$ & $1.59027[-11]$ & $1.9424[-06]$ & $2.2977[-12]$ & $3.13328[-17]$ \\
\hline 15 & $3.0478[+05]$ & $0,0,3$ & $5.763[-07]$ & $2.6892[-07]$ & $1.0747[-11]$ & $5.641[-07]$ & $4.553[-13]$ & $6.067[-18]$ \\
\hline 16 & $3.4731[+05]$ & $0,1,3$ & $5.537[-08]$ & $4.34051[-07]$ & $8.24267[-12]$ & $4.99474[-08]$ & $6.81358[-14]$ & $4.262735[-19]$ \\
\hline 17 & $3.9948[+05]$ & $0,0,4$ & $1.984[-08]$ & $1.91834[-07]$ & $6.25720[-12]$ & $1.95647[-08]$ & $1.132839[-14]$ & $1.229816[-19]$ \\
\hline 18 & $4.5262[+05]$ & $0,1,4$ & $3.509[-09]$ & $4.418[-07]$ & $4.832[-12]$ & $7.47[-09]$ & $3.394[-15]$ & $1.5381[-20]$ \\
\hline 19 & $4.8760[+05]$ & $0,0,5$ & $8.308[-10]$ & $1.54143[-07]$ & $4.20[-12]$ & $8.203[-10]$ & $3.815[-16]$ & $3.4612[-21]$ \\
\hline 20 & $5.3981[+05]$ & $0,1,5$ & $2.81[-10]$ & $2.7784[-07]$ & $3.4124[-12]$ & $2.6665[-10]$ & $2.2736[-16]$ & $9.2555[-22]$ \\
\hline 21 & $5.7996[+05]$ & $0,0,6$ & $6.305[-11]$ & $1.00364[-07]$ & $2.97056[-12]$ & $6.2577[-11]$ & $1.89062[-17]$ & $1.86371[-22]$ \\
\hline 22 & $6.3106[+05]$ & $0,1,6$ & $1.97[-11]$ & $1.7709[-07]$ & $2.5032[-12]$ & $1.920[-11]$ & $1.0330[-17]$ & $4.8522[-23]$ \\
\hline 23 & $6.6155[+05]$ & $0,0,7$ & $1.097[-11]$ & $9.3572[-08]$ & $2.28278[-12]$ & $1.08836[-11]$ & $3.0670[-18]$ & $2.49174[-23]$ \\
\hline 24 & $7.1590[+05]$ & $0,1,7$ & $2.053[-12]$ & $1.2881[-07]$ & $1.9470[-12]$ & $2.0136[-12]$ & $7.848[-19]$ & $3.9461[-24]$ \\
\hline 25 & $7.4009[+05]$ & $0,0,8$ & $2.696[-12]$ & $1.06801[-07]$ & $1.82286[-12]$ & $2.6584[-12]$ & $8.5706[-19]$ & $4.8688[-24]$ \\
\hline 26 & $7.9381[+05]$ & $0,1,8$ & $6.23[-13]$ & $7.8985[-08]$ & $1.5854[-12]$ & $6.174[-13]$ & $1.4690[-19]$ & $9.824[-25]$ \\
\hline 27 & $8.2166[+05]$ & $0,0,9$ & $1.22[-12]$ & $6.93[-08]$ & $1.48[-12]$ & 0 & 0 & 0 \\
\hline 28 & $9.5213[+05]$ & $0,0,10$ & $4.23[-12]$ & $1.11[-07]$ & $1.10[-12]$ & 0 & 0 & 0 \\
\hline 29 & $9.9872[+05]$ & $0,0,11$ & $8.85[-12]$ & $4.78[-08]$ & $1.002[-12]$ & 0 & 0 & 0 \\
\hline 30 & $1.0566[+06]$ & $0,0,12$ & $7.71[-09]$ & $1.15[-10]$ & $8.957[-13]$ & 0 & 0 & 0 \\
\hline 31 & $1.1095[+06]$ & $0,0,13$ & $8.50[-12]$ & $2.29[-09]$ & $8.123[-13]$ & 0 & 0 & 0 \\
\hline 32 & $1.1522[+06]$ & $0,0,14$ & $9.04[-12]$ & $1.70[-09]$ & $7.533[-13]$ & 0 & 0 & 0 \\
\hline 33 & $1.1984[+06]$ & $0,0,15$ & $9.64[-12]$ & $1.78[-09]$ & $6.963[-13]$ & 0 & 0 & 0 \\
\hline
\end{tabular}

connected in parallel. The actual Lorentzian form is obtained by dividing the effective impedance, $Z_{A}$, by $(-i \omega)$.

Since we can use complex impedance to represent a single Lorentzian, then it makes sense that we can use a circuit which showcases the difference between two impedances to simulate the difference between an absorbing Lorentzian and a reemitting one. A circuit known as the Wheatstone bridge does precisely this job; a schematic appears on the right side of Fig. 10.

The figure shows a comparison between a circuit analog for (a) the traditional, all-absorbers model and one for (b) our complete, classical model. Note that if $Z_{\mathrm{R}}$, the impedance representing the reemitters, ever equals zero, the circuit in Fig. 10(b) reverts to one that is equivalent to the traditional circuit in Fig. 10(a).

To add an absorbing component we simply add an assembly, in series, into the impedance blocks labeled $Z_{\mathrm{A}}$. To add a reemitting component we simply add an assembly in a similar fashion into the impedance blocks labeled $Z_{R}$. Because of the binary nature of the basis functions, every

nontrivial Lorentzian must fit into either block $Z_{\mathrm{A}}$ or $Z_{\mathrm{R}}$. No matter how many pairs of poles are used to represent a

(a)

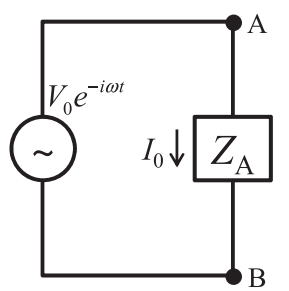

$V_{\mathrm{AB}}=I_{0}\left(\mathrm{Z}_{\mathrm{AB}}\right)$

$L_{\mathrm{AB}} \equiv \frac{Z_{\mathrm{AB}}}{-i \omega}=\frac{L_{\mathrm{A}}}{1-i \omega L_{\mathrm{A}} R_{\mathrm{A}}^{-1}-\omega^{2} L_{\mathrm{A}} C_{\mathrm{A}}}-\frac{L_{\mathrm{R}}}{1-i \omega L_{\mathrm{R}} R_{\mathrm{R}}^{-1}-\omega^{2} L_{\mathrm{R}} C_{\mathrm{R}}}$

FIG. 10. Circuit analog for (a) traditional model using only absorbing Lorentzians and (b) complete model using both absorbing and reemitting Lorentzians. 
single absorption or a single reemission, this circuit analog can still be applied, so long as the energy reemitted never exceeds the energy absorbed. Because the model includes all possible Lorentzians, absorbers as well as emitters, and includes all possible values of $Q$ (any finite real value $\geqslant 0$ ), we can now consider the model in Fig. 10(b) to be complete for any physically realizable material.

To finish the analog for susceptibility we need only to find the complex effective induction $L_{\mathrm{AB}}$ which is obtained by simply dividing the effective impedance $Z_{\mathrm{AB}}$ by $(-i \omega)$.

It is difficult to comment quantitatively on the validity of the model, since there is a definite spread in the measured data as well as the temperatures of the water samples used in Seglestein's compilation (in future work, temperature needs to be more consistent) but this fit appears to exemplify a unified model that can be performed without omitting an observed or interjecting an unobserved significant transition over the entire spectral region from dc to $1200 \mathrm{THz}(250 \mathrm{~nm})$. Since it was developed from fundamental principles of math and physics and not on any specific physical phenomena, the model is considered nonphenomenological.

\section{CONCLUSIONS}

Using analytic continuation, network control theory, and a more rigorous emphasis on physical realizability, it is indeed possible to derive a complete, analytic form to describe dispersion without assuming a physical model. The resultant model is therefore considered a priori or nonphenomenological. The present model is built entirely of pole-residue terms as expressed by (2) and (3) with the understanding that poles must appear in independent groups of two or more, their representations must possess causal-based symmetry, and the $\sigma_{n}$ residues of each group must sum to zero. Using a buildingblock approach, physics suggests that we only introduce poles in the simplest, physically allowed, and independent groups possible, the matched pole pairs, resulting in an all-Lorentzian dispersion model. Solitary Debye-type poles are simply not physically supported in the complete model. This dual-polebased model is concisely expressed in the frequency domain by (14) and in the time domain by (16) but with an alternative interpretation. The traditional Lorentzian-based model is the result of the mistaken assumption that every pole pair must describe absorption, which we now know to be overly restrictive. Some Lorentzians can in fact return energy, as evidenced by the ability of our complete model to accurately represent the broadband absorption spectrum of liquid water, including its optical valley, overcoming a significant physics mystery which has existed for close to 200 years.

With the possible exception of a simple pole at the origin, all poles must be below the real axis in the $\omega$ plane and all complex poles must be simple; only the purely imaginary poles can appear more than once. This model is complete in that the permittivity and permeability of any physically realizable material should fit this model from dc to daylight. (Note also that the dispersion parameters in this theory exhibit zero dispersion which means they can be entered without approximation into time-domain analysis.)
The Lorentzian model overcomes all the major shortcomings of the Debye model. In the time domain the Lorentzian kernels smoothly transition to zero as $t \rightarrow 0$. In the frequency domain this model correctly predicts the conductivity to display Debye-type dispersion. The Lorentzian expressions $\rightarrow 0$ at higher frequencies as $\omega^{-2}$. For magnetic materials the Lorentzian model not only allows the real component of susceptibility to be $<0$, it demands it; after all, since the initial response at $t=0^{+}$must change in the direction of the applied field, the time derivative in (4) must be positive, and so according to (4) $\chi(\omega) \rightarrow 0$ from the negative susceptibility direction.

An excellent example of a multiple-pole transition, which is allowed under the current theory, was reported in 2017 in a paper by Sehmi et al. [23]. In their paper the authors define a positive-whole-number parameter $D$ to describe the number of poles which interact with the dc conductivity pole at the origin in order to convey the independent Drude response. It is comforting to note that in their model, the dc conductivity pole is a simple pole and the sum of the residues is zero for any whole number $D \geqslant 1$. The authors, however, do not apply the same sum rule to their Lorentzian terms; instead, by following a recent trend by several other investigators in allowing the Lorentzian residues to be generally complex, they introduce physically unrealizable susceptibility which can become significant at two of the extremes of the dependent variables, time and frequency. This may be numerically acceptable over a limited band of interest but users need to be aware that this approach formally introduces a discontinuity and infinite slope (rendering negligible the assumed masses of the electrons in their metals) in the time-domain susceptibility at $t=0^{+}$. Furthermore, if even a single independent term such as this were to exist, it would result in $\omega^{-1}$ decay of the susceptibility in the frequency domain which would eventually dominate any other decay as $\omega \rightarrow \infty$ and this would be counter to our notions about inertia, the assumption that charges must have an associated mass, and the overwhelming evidence in support of $\omega^{-2}$ decay.

The all-Lorentzian model also gives insight into the mystery of why so many materials appear to fit the Debye model. With the understanding that all classically behaved transitions are probably made up of Lorentzian components, we have to conclude that the Debye susceptibility model is simply a local model approximation of the ultrawideband Lorentzian. In nature the rf quality factors tend to be in the WBL range, with $Q$ less than 0.5 . When we encounter such a transition, statistically speaking, it should come as no surprise that with several decades of potential values available to the $Q$ factors, that most of them should fail to fall in the relatively slender, WBL-measurable region between 0.3 and 0.5 .

Ultimately, the derivation in this paper helps explain why the phenomenological, Lorentzian, and Drude-Lorentzian models have withstood the test of time; have risen to a position of prominence among dispersion models; and have been so successful for over a century at matching observed dispersion in dielectric and in magnetic materials, with considerable accuracy. However, when more is required, then the guidelines 
set forth in this paper should allow investigators in the future to obtain that accuracy through the proper introduction of additional pole-residue terms as they are needed.

\section{ACKNOWLEDGMENT}

I wish to thank Dr. Rodolfo Enrique Diaz for many insightful exchanges and for personally handing me a copy of his paper [6] published in 1997 which started me on my enlightened journey into the physics behind dispersion of electromagnetic waves.

Any opinions, findings, and conclusions or recommendation expressed in this material are those of the author and such information must not be taken as establishing any contractual or other commitment binding upon the Rolls-Royce North American Technologies.
[1] J. Utz, Re: What percentage of the human body is composed of water? The MadSci Network, http://www.madsci.org/posts/ archives/2000-05/958588306.An.r.html (2000).

[2] H. Subramanian, P. Pradhan, Y. Liu, I. R. Capoglu, X. Li, J. D. Rogers, A. Heifetz, D. Kunte, H. K. Roy, A. Taflove, and V. Backman, Optical methodology for detecting histologically unapparent nanoscale consequences of genetic alterations in biological cells, Proc. Natl. Acad. Sci. USA 105, 20118 (2009).

[3] M. Rowan-Robinson, Night Vision: Exploring the Infrared Universe (Cambridge University Press, Cambridge, 2013), p. 23.

[4] A. K. Pierce, The infrared spectrum of the sun, Astron. Soc. Pacific Leafl. 9, 217 (1965), NASA Astrophysics Data System Bibcode .

[5] N. E. Dorsey, Properties of Ordinary Water-Substance in all its Phases (Reinhold, New York, 1940), pp. 330-331.

[6] R. E. Diaz and N. G. Alexopoulos, An analytic continuation method for the analysis and design of dispersive materials, IEEE Trans. Antennas Propag. 45, 1602 (1997).

[7] F. Shubitidze and U. Osterberg, Phenomenological model to fit complex permittivity data of water from radio to optical frequencies, Phys. Rev. E 75, 046608, (2007).

[8] D. W. Berreman and F. C. Unterwald, Adjusting poles and zeros to fit Restrahlen of $\mathrm{PrCl}_{3}$ and $\mathrm{LaCl}_{3}$, Phys. Rev. 174, 791 (1968).

[9] D. J. Segelstein, The complex refractive index of water, M.Sc. thesis, University of Missouri-Kansas City, 1981.

[10] A. Sihvola, Electromagnetic Mixing Formulas and Applications (The Institution of Engineering and Technology, London, UK, 1999), pp. 198-203.

[11] K. Heileman, J. Daoud, and M. Tabrizian, Dielectric spectroscopy as a viable biosensing tool for cell and tissue characterization and analysis, Biosens. Bioelectron. 49, 348 (2013).

[12] K. S. Cole and R. H. Cole, Dispersion and absorption in dielectrics, J. Chem. Phys. 9, 341 (1941).
[13] J. H. Van Vleck, Theory of Electric and Magnetic Susceptibilities (Oxford University Press, Oxford, 1932).

[14] P. Drude, Zur elektronentheorie der metalle, Ann. Phys. 306, 566 (1900).

[15] S. Havriliak and S. Negami, A complex plane representation of dielectric and mechanical relaxation processes in some polymers, Polymer 8, 161 (1967).

[16] M. E. Thomas, Optical Propagation in Linear Media: Atmospheric Gases and Particles, Solid-State Components and Water (Oxford University Press, Inc., Oxford, 2006).

[17] J. D. Jackson, Classical Electrodynamics, 2nd ed. (John Wiley \& Sons, New York, 1975), pp. 308-310.

[18] M. Y. Koledintseva, J. L. Drewniak, and X. Ye, Representation of gyromagnetic composite media for FDTD modeling, in Proceedings of IEEE International Symposium on Electromagnetic Compatibility (IEEE, Piscataway, NJ, 2001), pp. 555-558.

[19] W. H. Press, S. A. Teukolsky, W. T. Vetterling, and B. P. Flannery, Numerical Recipes in C, the Art of Scientific Computing, 2nd ed. (Cambridge University Press, New York, 1997), pp. 683-688.

[20] J. D. Mason, M. T. Cone, and E. S. Fry, Ultraviolet (250$550 \mathrm{~nm}$ ) absorption spectrum of pure water, Appl. Opt. 55, 7163 (2016).

[21] J. B. Hasted, Aqueous Dielectrics, Studies in Chemical Physics (Chapman and Hall, London, 1973), p. 61.

[22] M. N. Afsar and J. B. Hasted, Measurements of the optical constants of liquid $\mathrm{H}_{2} \mathrm{O}$ and $\mathrm{D}_{2} \mathrm{O}$ between 6 and $450 \mathrm{~cm}^{-1}$, J. Opt. Soc. Am. 67, 902 (1977).

[23] H. S. Sehmi, W. Langbein, and E. A. Muljarov, Optimizing the Drude-Lorentz model for material permittivity: Method, program, and examples for gold, silver, and copper, Phys. Rev. B 95, 115444 (2017), p. 2, Eq. (3). 